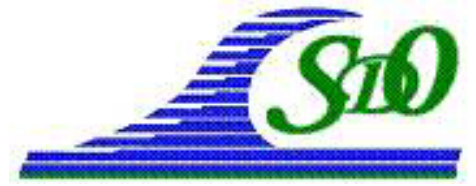

XI ìmes Journées Nationales Génie Côtier - Génie Civil

Les Sables d'Olonne, 22-25 juin 2010

DOI:10.5150/jngcgc.2010.088-M C Editions Paralia CFL

disponible en ligne - http://www.paralia.fr - available online

\title{
Caractérisation expérimentale du sillage généré par une hydrolienne. Influence du taux de turbulence ambiant
}

\author{
Fabrice MAGANGA $^{1,2}$, Grégory GERMAIN ${ }^{2,3}$, Jean-Valery FACQ ${ }^{2,3}$, \\ Benoit GAURIER $^{2,3}$, Elie RIVOALEN ${ }^{4}$, Grégory PINON ${ }^{1}$
}

1. LOMC, FRE 3102 CNRS, Univ. Le Havre, France.

2. IFREMER, Serv. Hydrodynamique \& OcéanoMétéo, 62200 Boulogne/Mer, France.

3. Univ. Lille Nord de France, 59000 Lille, France.

4. INSA de Rouen, LMR, F-76801 Saint-Etienne du Rouvray, France.

gregory.germain@ifremer.fr

\section{Résumé :}

Le fonctionnement d'une hydrolienne est régi par les caractéristiques de l'écoulement incident. Cet article présente les résultats d'une étude expérimentale visant à déterminer les effets du taux de turbulence de l'écoulement ambiant sur le fonctionnement d'une hydrolienne et sur le sillage généré.

Les essais expérimentaux ont été réalisés dans la veine hydrodynamique à surface libre de l'Ifremer, dont on peut faire varier le taux de turbulence de l'écoulement de 5 à $25 \%$. Le prototype utilisé est une turbine tri-pales à axe horizontal de $0,7 \mathrm{~m}$ de diamètre (échelle 1/30). Des cartes de vitesses moyennes, de taux de turbulence, mais également d'énergie cinétique turbulente et de contrainte de cisaillement ont été obtenues à partir de mesures par vélocimétrie laser pour une vitesse de l'écoulement incident de $0,8 \mathrm{~m} / \mathrm{s}$. L'analyse des sillages proche et lointain a été effectuée : le sillage proche est caractérisé par un fort gradient de cisaillement et une intensité de turbulence élevée, alors que le sillage lointain est caractérisé par son expansion. Une réduction du sillage d'un facteur 3 cumulée à d'importantes fluctuations d'efforts ont également pu être observées pour les taux de turbulence les plus élevés.

\section{Mots-clés :}

Hydrolienne - Mesures LDV - Essais expérimentaux - Effet de la turbulence - Energie marine

\section{Introduction}

Un nombre croissant de pays cherchent à augmenter la part de leur production d'énergie issue des énergies renouvelables. L'exploitation des courants marins en est un des moyens, cette ressource d'énergie marine étant particulièrement élevée en Europe, et notamment en France et dans les DOM/ROM. Cependant, les technologies nécessaires pour valoriser ce potentiel ne sont pas matures. Elles ont besoin d'être accompagnées pour réellement émerger et leurs impacts environnementaux sont relativement peu connus malgré de récentes avancées. 
De nombreux concepts de système de récupération de l'énergie des courants marins sont actuellement en cours de développement et d'expérimentation (GERMAIN, 2008). Le comportement et le rendement de ce type de machines sont aujourd'hui encore déterminés pour des conditions bien spécifiques de fonctionnement, i.e. en présence d'un écoulement incident idéalisé (uniforme et stationnaire). Certains travaux visent à étendre ces études à des conditions de fonctionnement moins spécifiques : interaction avec la surface libre, profil de vitesse vertical (MYERS \& BAHAJ, 2008). Malgré cela, de nombreuses questions restent aujourd'hui en suspens. Nous proposons ici d'apporter des éléments de réponses pour l'une d'entre elles : quel est l'effet du taux de turbulence ambiant sur le fonctionnement d'une hydrolienne et sur les caractéristiques de son sillage?

Les résultats présentés sont issus des essais expérimentaux réalisés dans la veine hydrodynamique à surface libre de l'Ifremer, dont on peut faire varier le taux de turbulence de l'écoulement (typiquement entre 5 et $25 \%$ ). La maquette utilisée est une turbine tri-pales à axe horizontal de diamètre $\mathrm{D}=0,7 \mathrm{~m}$ (échelle 1/30), (GERMAIN et al., 2007). Des considérations sur le rendement sont effectuées, mais l'essentiel des résultats permet de quantifier l'influence du taux de turbulence de l'écoulement amont sur les caractéristiques du sillage généré par la turbine. Les mesures ont été effectuées à l'aide d'un système de vélocimétrie laser Doppler 2D en un nombre conséquent de profils. Ainsi, les effets du taux de turbulence de l'écoulement ambiant sur les caractéristiques du sillage sont analysés à partir des cartes de vitesses moyennes et de taux de turbulence (les cartes d'énergie cinétique turbulente et de contrainte de cisaillement seront présentées lors de la présentation orale).

\section{Approche expérimentale}

L'étude expérimentale de ce travail a été effectuée dans le bassin à houle et courant de l'Ifremer, Boulogne/Mer (figure 1). Dans cette installation, le taux de turbulence de l'écoulement peut être adapté par l'introduction de grilles perforées en amont de la section d'essais. Les valeurs de taux de turbulence considérées pour cette étude sont : 5 , 8 et $25 \%$, ce qui correspond à des niveaux pouvant être rencontrés dans des zones à plus ou moins forts courants (MASTERS et al., 2007 ; GUINOT \& LE BOULLUEC, 2008). Le taux de turbulence est déterminé de la manière suivante :

$T I=100 \times \frac{\sqrt{(\sigma u)^{2}(\sigma v)^{2}+(\sigma w)^{2}}}{\sqrt{u^{2}+v^{2}+w^{2}}}, u, v$ et $w$ correspondant aux composantes de vitesses selon les trois directions principales.

La maquette utilisée est une turbine tri-pales à axe horizontal de $0,7 \mathrm{~m}$ de diamètre (échelle 1/30), (GERMAIN et al., 2007). La veine présentant une section d'essais de 4 $\mathrm{m}$ de large, $2 \mathrm{~m}$ de haut sur une longueur utile de $18 \mathrm{~m}$, le blocage de la section engendré par la structure est de 5\%. La vitesse de l'écoulement incident est fixée pour 


\section{XI $I^{\text {emes }}$ Journées Nationales Génie Côtier - Génie Civil}

Les Sables d'Olonne, 22-25 juin 2010

cette étude à $0,8 \mathrm{~m} / \mathrm{s}$ et le TSR (Tip Speed Ratio, vitesse de rotation divisée par la vitesse de l'écoulement incident) à 6 . Considérant le diamètre de la turbine comme longueur caractéristique du système, le nombre de Reynolds caractérisant l'écoulement est donc relativement élevé : $R e=5,6 \times 10^{6}$.

Dans le but de quantifier l'influence du taux de turbulence sur les caractéristiques du sillage généré par la turbine, un nombre conséquent de profils de vitesses est effectué à l'aide d'un système de vélocimétrie laser Doppler 2D (l'écoulement étant ensemencé à l'aide de micro-billes de verre argentées de 10 microns). Les profils ont été effectués tous les diamètres jusqu'à une distance de 10D, chacun d'entre eux étant constitué de 25 points de mesures.
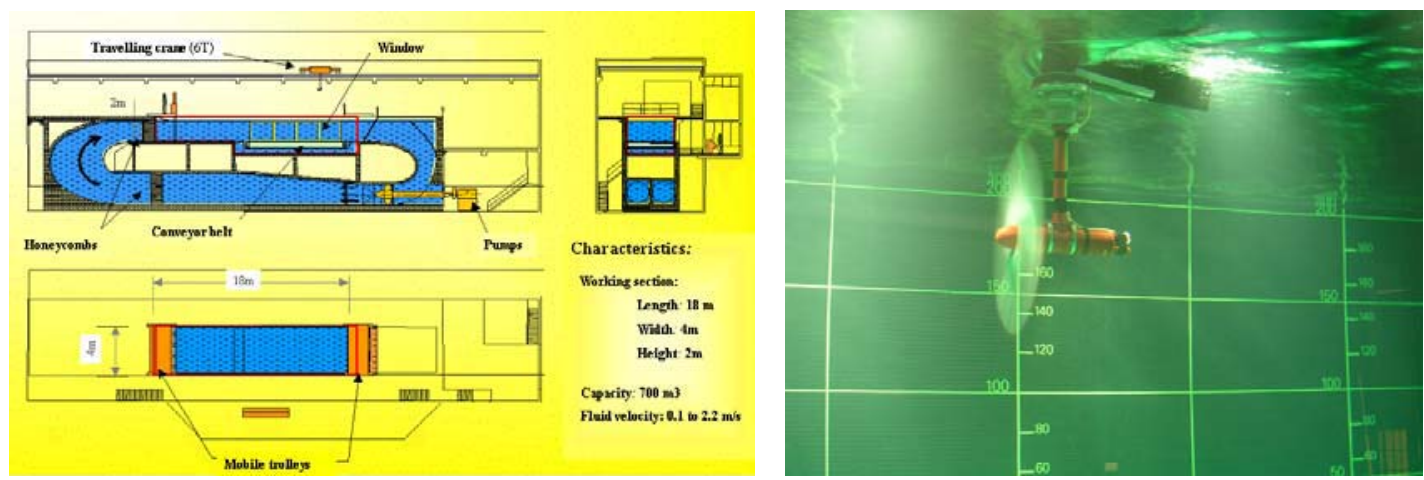

Figure 1. Caractéristiques du bassin à houle et courant de l'Ifremer et maquette d'hydrolienne en fonctionnement.

\section{Analyse des résultats}

Le sillage d'une turbine comporte deux parties aux caractéristiques et effets distincts : le sillage proche et le sillage lointain. Le premier est caractéristique des performances de la machine et du processus de captage d'énergie, alors que la quantification du sillage lointain est primordiale pour optimiser le positionnement d'hydroliennes placées à proximité les unes des autres, les turbines positionnées en aval étant soumises à un écoulement plus perturbé (plus faible et plus turbulent, (MYERS \& BAHAJ, 2009)).

De manière à quantifier l'influence du taux de turbulence de l'écoulement incident sur le comportement d'une hydrolienne, nous avons effectué des mesures de sillage pour trois taux de turbulence : 5,8 et $25 \%$. Les champs de vitesses correspondant sont présentés sur la figure 2 (issus de mesures LDV dans le plan horizontal de l'axe principal de la turbine). 

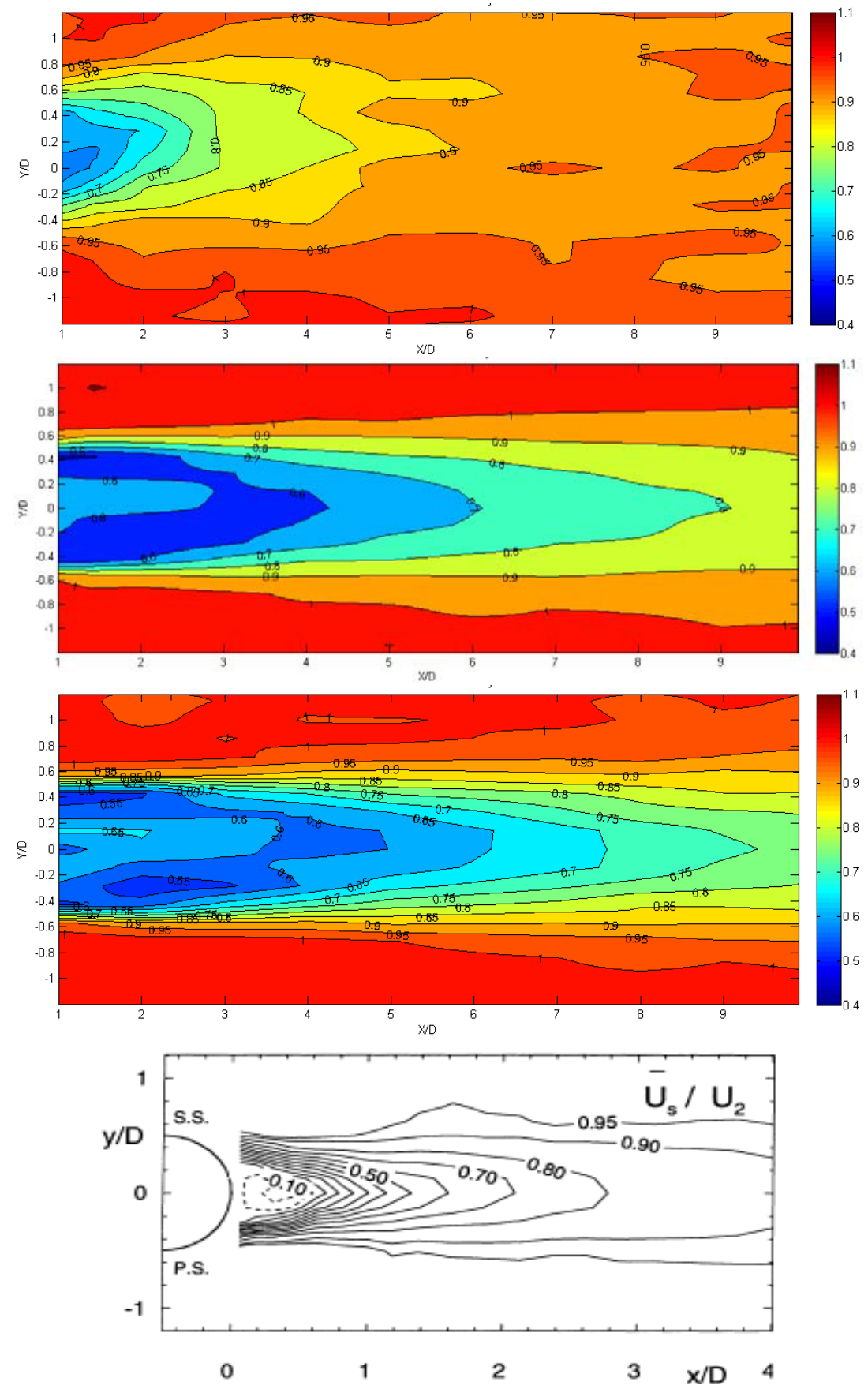

Figure 2. Evolution du déficit de vitesse moyenne dans le sillage de la turbine pour trois taux de turbulence : respectivement 25,8 et $5 \%$ de haut en bas, à TSR=6, comparés aux données issues de UBALDI \& ZUNINO (2000).

Ces champs permettent de caractériser l'expansion et le déficit de vitesse dans le sillage de la turbine pour chaque taux de turbulence de l'écoulement amont. Le retour à une vitesse correspondant à $80 \%$ de la vitesse incidente s'effectue trois fois plus rapidement pour un taux de turbulence de $25 \%$ que pour un taux de turbulence de $8 \%$ (alors que 
cette valeur n'est toujours pas retrouvée à une distance de $10 \mathrm{D}$ pour un taux de turbulence de $5 \%$ ). Les effets de sillage sont négligeables après seulement $5 \mathrm{D}$ dans la cas où $\mathrm{TI}=25 \%$ alors qu'ils restent conséquents dans les deux autres cas. L'extension transverse du sillage est quant à elle d'autant plus large que le taux de turbulence de l'écoulement incident est élevé. Ces résultats sont en accord avec les considérations généralement effectuées dans le cas d'une sphère, d'un cylindre (ADRARAMOLA et al., 2006) ou d'une éolienne (VERMEER et al., 2003).

La comparaison (figure 2) entre l'évolution du sillage issu d'une hydrolienne et celui issu d'une éolienne (UBALDI \& ZUNINO, 2000) montre que l'expansion du sillage suivant la direction de l'écoulement incident est trois fois plus rapide pour une éolienne que pour une hydrolienne : un déficit de vitesse de $20 \%$ est présent jusqu'à $9 \mathrm{D}$ dans le cas d'une hydrolienne, alors qu'il disparaît avant $3 \mathrm{D}$ dans le cas d'une éolienne.

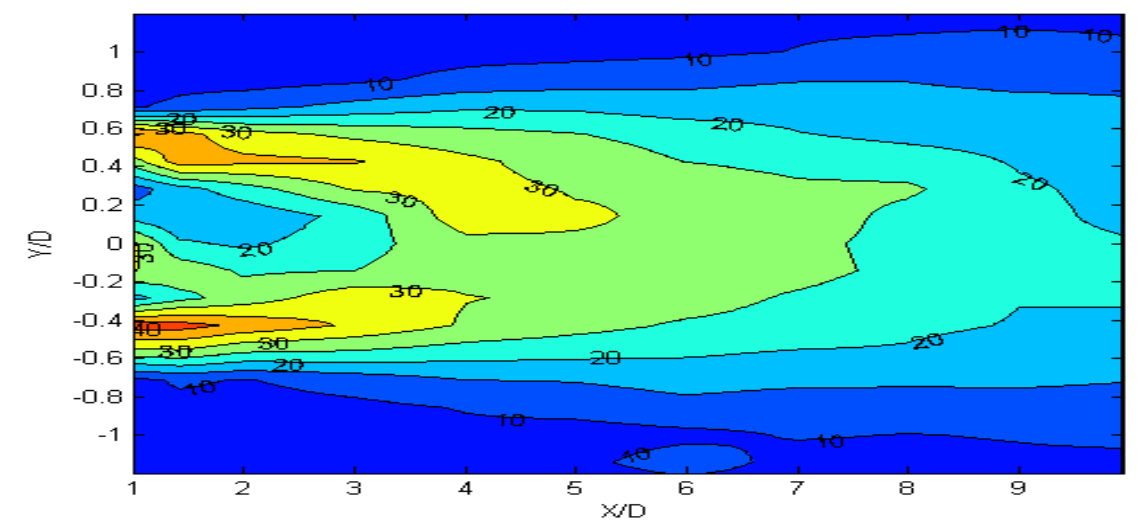

Figure 3. Evolution du taux de turbulence dans le sillage de la turbine $T S R=6, T I=8 \%, U=0.8 \mathrm{~m} / \mathrm{s}$.

L'évolution du taux de turbulence dans le sillage de la turbine est présentée sur la figure 3. Dans le sillage proche, un taux de turbulence de plus de 35\% est observé dans la zone d'émission tourbillonnaire en bout de pales. Cette zone est perceptible à plus de $5,5 \mathrm{D}$ et un taux de turbulence de l'ordre de $20 \%$ est rencontré jusqu'à plus de $9 \mathrm{D}$, sur une largeur d'environ 1,5 D. L'analyse des performances de la machine (MAGANGA et al., 2009) montre que ce type de perturbations peut avoir des conséquences non négligeables sur le fonctionnement d'hydroliennes pouvant se trouver dans le sillage d'une machine positionnée en amont.

Six profils de vitesses axiales moyennes sont reportés sur la figure 4 pour les trois taux de turbulence de l'écoulement amont : 5, 8 et $25 \%$. Ces profils montrent l'évolution du déficit de vitesse dans le sillage pour l'ensemble des conditions d'entrée testées. L'émission tourbillonnaire de bout de pales est ici aussi bien marquée jusqu'à $\mathrm{X} / \mathrm{D}=3$ pour $\mathrm{TI}=8 \%$. Ce phénomène est masqué à $\mathrm{TI}=25 \%$ du fait de la décroissance rapide $\mathrm{du}$ déficit de vitesse dans le sillage (reporté sur la figure 5). Dans le sillage lointain, 
démarrant à $\mathrm{X} / \mathrm{D} \sim 2$ pour $\mathrm{TI}=25 \%$ et $\mathrm{X} / \mathrm{D} \sim 5$ pour $\mathrm{TI}=8 \%$, la répartition classique sous forme de Gaussienne est observée. L'évolution de la vitesse axiale moyenne le long de l'axe de symétrie de la turbine à $\mathrm{TI}=8 \%$ est équivalente à celle reportée dans (MYERS \& BAHAJ, 2009), mais avec des valeurs plus basses (de l'ordre de 10\%).
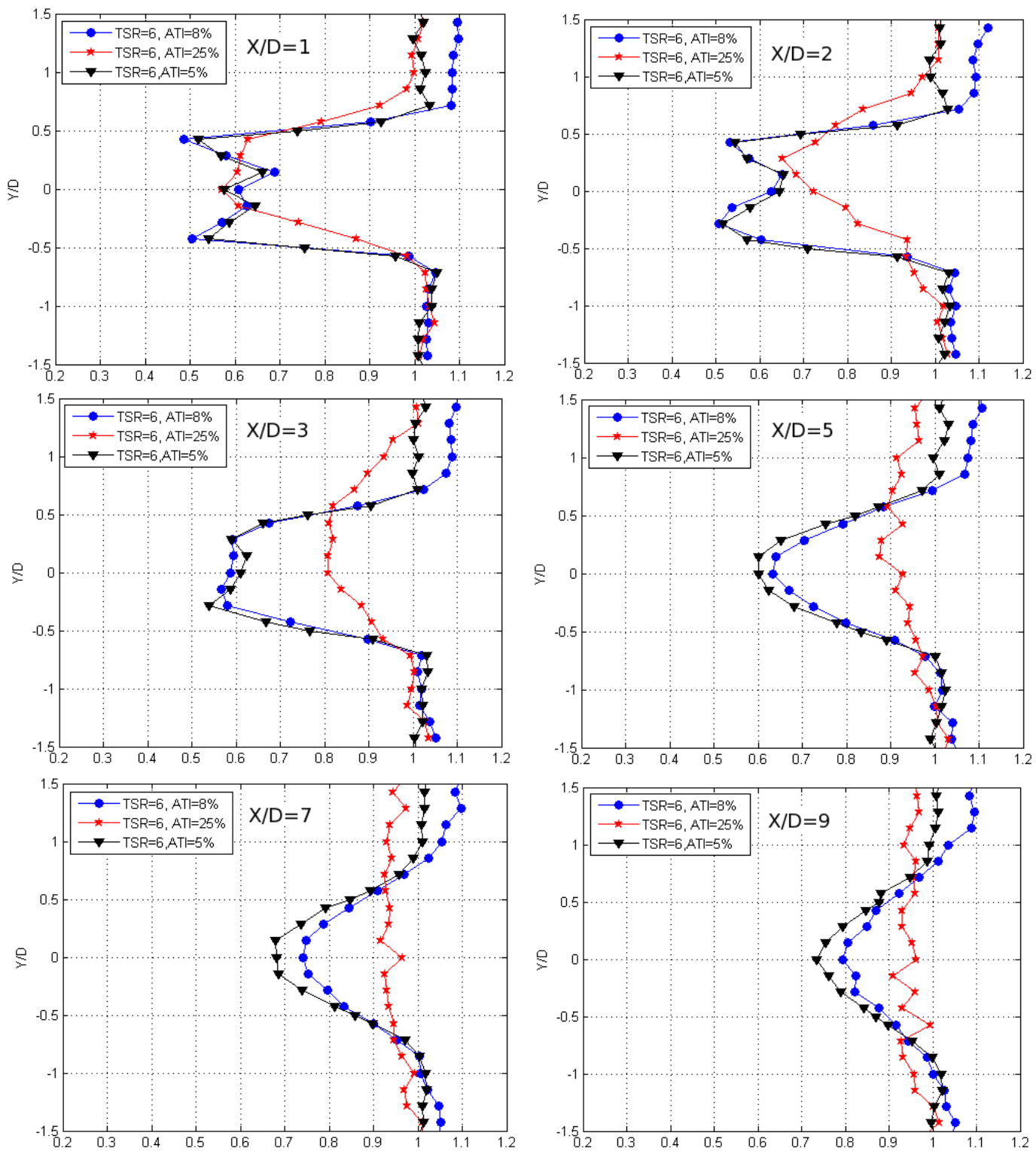

Figure 4. Profils de vitesses axiales moyennes pour les trois niveaux de turbulences considérés, à $T S R=6$ et $X / D=1$ à 9 .

L'augmentation du taux de turbulence de l'écoulement incident a des effets non seulement sur le sillage généré par l'hydrolienne, mais également sur son fonctionnement mécanique, comme on peut le voir sur la figure 6 , où l'évolution du coefficient de traînée est donnée pour $\mathrm{TI}=5,8$ et $25 \%$. Un écart entre 3 et $9 \%$ sur le 
coefficient de traînée est obtenu suivant le type d'écoulement considéré. Des effets non négligeables sur les fluctuations d'efforts sont également perceptibles : les fluctuations d'efforts sont doubles pour $\mathrm{TI}=25 \%$, comparés à celles obtenues pour $\mathrm{TI}=5 \%$. Une étude spécifique sur le comportement structurel des pales et la fatigue des matériaux utilisés est nécessaire pour quantifier plus finement ces effets.

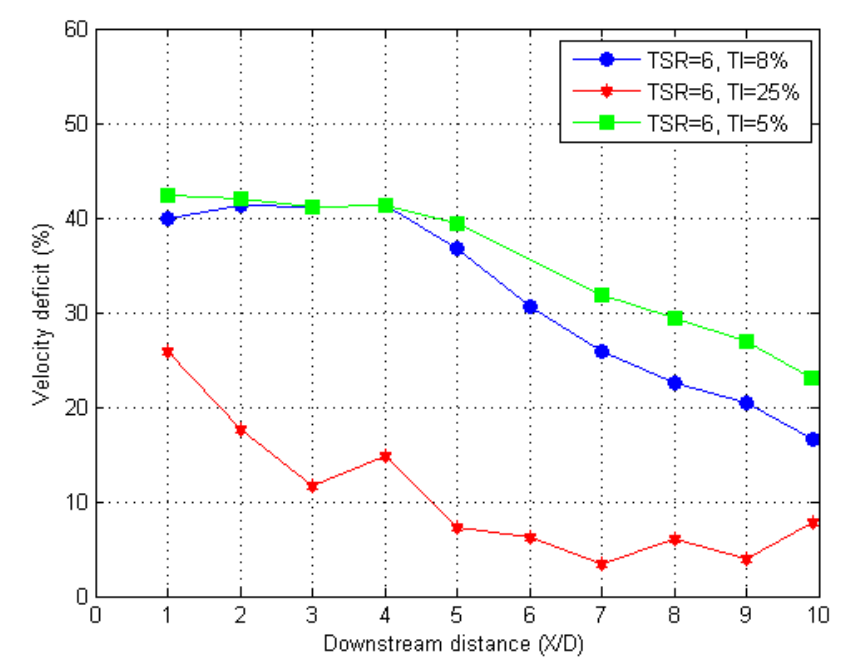

Figure 5. Evolution du déficit de vitesse à $T S R=6$.

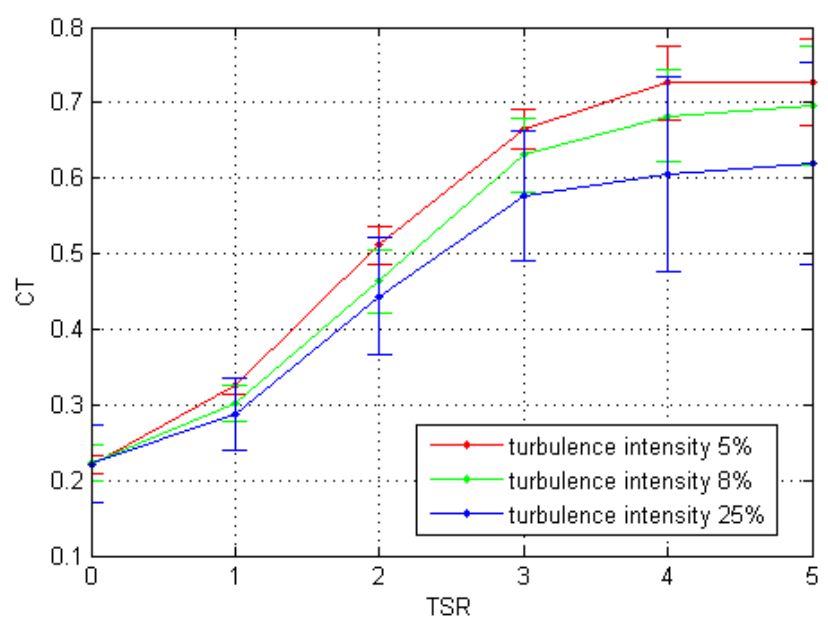

Figure 6. Coefficients de traînée pour les trois taux de turbulence.

\section{Conclusions}

Les résultats présentés ont permis de caractériser l'influence du taux de turbulence de l'écoulement amont sur le comportement et l'évolution du sillage généré par une hydrolienne tri-pales à axe horizontal. Le sillage proche est caractérisé par un fort gradient de cisaillement et une intensité de turbulence élevée, alors que le sillage lointain est caractérisé par son expansion. Une réduction du sillage d'un facteur 3 et 
d'importantes fluctuations d'efforts ont pu être observées pour les taux de turbulence les plus élevés.

Ces résultats constituent une base de données idéale pour la validation des outils numériques développés en parallèle du travail expérimental présenté dans ce document (MAGANGA et al., 2008). Ces développements seront étendus à des conditions d'utilisation plus réalistes, notamment par la prise en compte des effets combinés de la houle et du courant. Une attention particulière devra être portée sur les effets tridimensionnels des sillages émis par les hydroliennes sur l'environnement physique immédiat et plus particulièrement sur leur impact sédimentaire et les phénomènes d'interaction entre hydroliennes placées en espace proche.

\section{Références bibliographiques}

ADRARAMOLA M.S., AKINLADE O.G., SUMNER D., BERGSTROM D.J., SCHENSTEAD A.J. (2006). Turbulence wake of a finite circular cylinder of small aspect ratio. Journal of Fluids and Structure, Vol.22, Issue 6-7, pp 919-928. doi:10.1016/j.jfluidstructs.2006.04.007

GERMAIN G. (2008). Marine current energy converter tank testing practices. ICOE 2008, Brest.

GERMAIN G., BAHAJ A.S., ROBERTS P., HUXLEY-REYNARD C. (2007). Facilities for marine current energy converter characterization. 7th EWETEC, Porto.

GUINOT F., LE BOULLUEC M. (2008). Realistic marine flow conditions for current turbines studies. ICOE 2008, Brest.

MAGANGA F., PINON G., GERMAIN G., RIVOALEN E. (2008). Numerical Simulation of the wake of marine Current Turbine with a particle method. WREC X, Glasgow.

MAGANGA F., GERMAIN G., KING J., PINON G., RIVOALEN E. (2009). Experimental study to determine flow characteristic effects on marine current turbine behaviour. $8^{\text {th }}$ EWTEC, Upsala.

MASTERS I., ORME J.A.C., CHAPMAN J. (2007). Towards realistic marine flow conditions for tidal stream turbine. $7^{\text {th }}$ EWETEC, Porto.

MYERS L., BAHAJ A.S. (2008). The effect of boundary proximity upon the wake structure of horizontal axis marine current turbines. $27^{\text {th }}$ OMAE, Estoril.

MYERS L., BAHAJ A.S. (2009). Near wake properties of horizontal axis marine current turbines. $8^{\text {th }}$ EWTEC, Upsala.

UBALDI M., ZUNINO P. (2000). An experimental study of the unsteady characteristics of the near wake of a turbine blade. Exp. Thermal \& Fluid Science, Vol. 23, pp 23-33. doi:10.1016/S0894-1777(00)00030-3

VERMEER L.J., SORENSEN J.N., CRESPO A. (2003). Wind turbine wake aerodynamics. Progressing in Aerospace Sciences, Vol. 39, pp 467-510. doi:10.1016/S0376-0421(03)00078-2 